\title{
Hepatic population derived from human pluripotent stem cells is effectively increased by selective removal of undifferentiated stem cells using YM155
}

Seok-Jin Kang, Young-Il Park, So-Ryeon Hwang, Hee Yi, Nga Tham, Hyun-Ok Ku, Jae-Young Song and Hwan-Goo Kang*

\begin{abstract}
Background: Pluripotent stem cells (PSCs) such as embryonic stem cells and induced pluripotent stem cells are promising target cells for cell regenerative medicine together with recently advanced technology of in-vitro differentiation. However, residual undifferentiated stem cells (USCS) during in-vitro differentiation are considered a potential risk for development of cancer cells and nonspecific lineage cell types. In this study we observed that USCS still exist during hepatic differentiation, consequently resulting in poor quality of the hepatic population and forming teratoma in vivo. Therefore, we hypothesized that effectively removing USCs from in-vitro differentiation could improve the quality of the hepatic population and guarantee safety from risk of teratoma formation.

Methods: Human PSCs were differentiated to hepatocytes via four steps. YM155, a known BIRC5 inhibitor, was applied for removing the residual USCs on the hepatic differentiation. After YM155 treatment, hepatocyte development was evaluated by measuring gene expression, immunostaining and hepatic functions at each stage of differentiation, and forming teratomas were confirmed by cell transplantation with or without YM155.

Results: The selected concentrations of YM155 removed USCs (NANOG ${ }^{+}$and $\mathrm{OCT}_{4}^{+}$) in a dose-dependent manner. As a result, expression of endodermal markers (SOX17, FOXA2 and CXCR4) at stage II of differentiation and hepatic markers (ALB, AFP and HNF4A) at stage III was up-regulated by YM155 treatment as well as the hepatic population $\left(A L B^{+}\right.$), and functions (ALB/urea secretion and CYP450 enzyme activity) were enhanced at the final stage of differentiation (stage IV). Furthermore, we demonstrated that NANOG and OCT4 expression remaining until stage III (day 15 of differentiation) completely disappeared when treated with YM155 and teratoma formation was effectively prevented by YM155 pretreatment in the in-vitro study.
\end{abstract}

Conclusions: We suggest that the removal of USCS using YM155 could improve the quantity and quality of induced hepatocytes and eliminate the potential risk of teratoma formation.

Keywords: Pluripotent stem cells, Hepatic differentiation, Residual undifferentiated stem cell, YM155

\footnotetext{
* Correspondence: kanghg67@korea.kr

Vet Drugs and Biologics Division, Animal and Plant Quarantine Agency, 177,

Hyeoksin 8-ro, Gimcheon-si, Gyeongsangbuk-do 39660, Republic of Korea
} 


\section{Background}

Human pluripotent stem cell (PSC)-induced hepatocytes (iHeps) are a promising target for drug development and cell transplantation. Over the past 10 years, many protocols for hepatocyte induction from embryonic stem cells (ESCs) and induced pluripotent stem cells (iPSCs) have been developed by recapitulating in-vivo hepatic development [1-6]. Acquiring pure hepatocytes is a crucial factor for hepatotoxicity screening of drug candidates and stem cell-derived hepatocyte therapy. Thus far, hepatocyte induction has enabled progress in acquiring highly purified hepatocytes and in enhancing hepatic functions [3, 7-9].

There are many previous reports about undifferentiated stem cells (USCs) that remain during in-vitro differentiation. The residual USCs exist long term in both in-vitro and in-vivo differentiation [10]. This is a considerably critical concern in cell replacement therapy because the residual stem cells have inherent problems (i.e., teratoma formation) [11-13]. Therefore, eliminating the residual USCs is an indispensable step for safer cell therapy and efficient hepatotoxicity screening of drug candidates. Recently, selective removal of USCs has been tried to prevent tumorigenic potential and teratoma formation using antibodybased strategy in clinical fields [14-16]. In addition, small molecules have been suggested to effectively eliminate the risk of teratomas $[17,18]$. Of these molecules, YM155 is one of the inhibitors targeting the BIRC5 (Survivin) gene, an anti-apoptotic factor, which is highly overexpressed in ESCs/iPSCs and cancer stem cells. Thus, this molecule can induce selective apoptotic cell death of USCs [18]. Currently, the compound has been introduced in clinical applications for cancer therapy and interruption of teratoma formation after stem cell therapy [19].

In our former study, USCs remained in a small portion during hepatic differentiation and consequently developed nonhepatic phenotypes [20]. Lee et al. [18] reported that YM155 effectively eliminated USCs without affecting differentiated counterparts. However, little research has been conducted to selectively remove the undifferentiated cells during in-vitro hepatic differentiation. In the present study, we investigated the effects of YM155 on hepatic differentiation using human PSCs concerning the effective treatment stage of differentiation and optimal concentration of YM155 and in-vivo teratoma formation. To our knowledge, this is the first report of applying YM155 to in-vitro hepatic differentiation.

\section{Methods}

\section{Cell culture}

Human ESCs (WA01) and iPSCs (iPS(IMR90)-1) were purchased from the WiCell Research Institute (WI, USA; http://wicell.org/). Human iPSCs (QIA7) were established from adipose tissue-derived stromal cells in our laboratory
[21]. These cells were mechanically passaged using Dispase (STEMCELL Technologies, Canada) and maintained on a Matrigel-coated plate (BD Bioscience, CA, USA) with mTeSR1 medium (STEMCELL Technologies). HepG2 (ATCC, VA, USA) was cultured in EMEM (ATCC) containing 10\% FBS (Gibco-BRL, NY, USA) and 1\% penicillin and streptomycin (Millipore, MA, USA). Human cryopreserved primary hepatocytes ( $\mathrm{p}$-Heps) were purchased as BD Gentest ${ }^{\text {Ts }}$ Inducible-Qualified Human CryoHepatocytes (Lot \#178; BD Bioscience). Human hepatocytes were cultured in HMM medium using a SingleQuots ${ }^{\mathrm{TM}}$ kit (Lonza, MD, USA) on Coning BioCoat Collagen I-coated plates (BD Bioscience). Human adipose tissue-derived stromal cells (hAT-SCs) were maintained with DMEM containing 10\% FBS, $50 \mathrm{U} / \mathrm{ml}$ penicillin and $50 \mu \mathrm{g} / \mathrm{ml}$ streptomycin.

\section{Differentiation of PSCs into hepatocytes}

For the hepatic differentiation of human PSCs, a previously reported hepatic differentiation protocol designed for human ESCs [1] was applied with some modifications. Briefly, human PSCs were plated at a density of $2.5 \times 10^{5}$ cells $/ \mathrm{ml}$ on Matrigel-coated six-well plates with mTeSR1 medium (STEMCELL Technologies) including ROCK inhibitor (Y27632; STEMCELL Technologies). The medium was replaced with definitive endodermal induction medium (DE) for 5 days (stage I). The DE medium consisted of RPMI 1640 (without L-glutamine; Gibco-BRL) supplemented with $2 \mathrm{mM}$ L-glutamine (Millipore), $0.5 \mathrm{mg} / \mathrm{ml}$ albumin fraction V (Merck Millipore, Germany) and $100 \mathrm{ng} / \mathrm{ml}$ Activin A (PeproTech, NJ, USA). Afterward, the definitive endodermal cells were differentiated into hepatoblasts using the hepatic endodermal medium (Hep-1) for 5 days (stage II) followed by hepatic specification medium (Hep-2) for 5 days (stage III). Hep-1 and Hep-2 comprised the HBM SingleQuots $^{\text {TM }}$ kit (Lonza) supplemented with $30 \mathrm{ng} / \mathrm{ml}$ FGF4 (PeproTech) and $20 \mathrm{ng} / \mathrm{ml} \mathrm{BMP2} \mathrm{(Invitrogen,} \mathrm{MD,} \mathrm{USA)}$ at stage II and $20 \mathrm{ng} / \mathrm{ml} \mathrm{HGF} \mathrm{(PeproTech)} \mathrm{and} 20 \mathrm{ng} / \mathrm{ml}$ OSM (R\&D Systems, MN, USA) at stage III, respectively. After hepatic specification, the cells were further matured using the HMM SingleQuots ${ }^{\mathrm{TM}}$ kit for 5 days (stage IV).

\section{RT-PCR}

Total RNA was isolated from the cells using the RNeasy mini kit (Qiagen, Germany). cDNA was synthesized from $1 \mu \mathrm{g}$ of total RNA primed with AcuPower cDNA synthesis premix (Bioneer, Republic of Korea). Primer sets are represented in Additional file 1: Table S1. Realtime PCR was performed using the CFX (Bio-Rad, CA, USA) instrument and the SYBR Green script premix (Bio-Rad). Normalization of samples was determined by GAPDH, and all sets of reactions were conducted in triplicate $(n=3)$. The relative expression levels are expressed as a fold-change of the indicated control. 


\section{Immunocytochemistry}

For immunostaining, the cells were fixed with $4 \%$ paraformaldehyde (PFA; Thermo Scientific) for 5 min after washing with $1 \times$ phosphate-buffered saline (PBS). After rinsing with $1 \times$ rinse buffer $(1 \times$ Tris $-\mathrm{HCl}$ including $0.05 \%$ Tween20 ), permeabilization was performed with $0.1 \%$ Triton $X-$ 100 for $10 \mathrm{~min}$. After blocking with blocking solution (1:20 diluent with $1 \times$ PBS) for $30 \mathrm{~min}$ at room temperature, each primary antibody was reacted by incubating overnight at $4{ }^{\circ} \mathrm{C}$. After rinsing, secondary antibodies were applied in incubation for $1 \mathrm{~h}$ at room temperature. Information for antibodies is listed in Additional file 1: Table S2. The nuclei were stained for $1 \mathrm{~min}$ with Hoechst33258 (Invitrogen) diluted in 1× PBS (1:10,000). Immunofluorescence was detected under a fluorescence microscope (Axiovert; Carl Zeiss, Germany).

\section{Cytotoxicity assay}

To measure cytotoxicity by YM155 treatment, QIA7 (stage 0), QIA7-iHeps (stage I and stage II) and hAT-SCs were seeded on Matrigel-coated 96-well plates $\left(2 \times 10^{4}\right.$ cells per well) and cultured for $24 \mathrm{~h}$ at $37{ }^{\circ} \mathrm{C}$. Serial concentrations of YM155 $(0-100 \mu \mathrm{M})$ were diluted with each culture medium as already described and incubated with the cells for $24 \mathrm{~h}$. The culture medium was subsequently replaced with $100 \mu \mathrm{l}$ of each fresh medium containing $10 \%$ (v/v) 2-(4-Iodophenyl)-3-(4-nitrophenyl)-5-(2,4-disulfophenyl)-2H-tetraxolium (WST) reagent (DoGEN, Republic of Korea) and again incubated for $2 \mathrm{~h}$ at $37^{\circ} \mathrm{C}$. Absorbance was read using a luminometer (FlexStation III; Molecular Device, CA, USA) at $450 \mathrm{~nm}$, and the results were expressed as the percent of nontreated control.

\section{Caspase-3 activity}

To detect apoptotic cell death, we used the Caspase-3 colorimetric activity assay kit (Millipore). QIA7, QIA7iHeps (day 7 of differentiation) and hAT-SCs were seeded with a relevant cell number and incubated overnight at $37^{\circ} \mathrm{C}$. Each cell was treated by serial concentrations of YM155 (1-100 nM) for $16 \mathrm{~h}$. After lysis with $1 \times 10^{6}$ cells, the supernatant was incubated with Caspase-3 substrate for $1 \mathrm{~h}$ at $37^{\circ} \mathrm{C}$. Absorbance was read using a luminometer (FlexStation III) at $405 \mathrm{~nm}$, and the activities were expressed as a fold-increase against DMSO control.

\section{Flow cytometry}

Cells were dissociated in 0.05\% Trypsin-EDTA (Invitrogen) and then resuspended in 10\% FBS/DMEM (v/v). The collected cells were fixed with 4\% PFA, permeabilized with $0.1 \%$ Triton $\mathrm{X}-100$ and stained with an antibody against OCT4 and Albumin. The analyses were performed using FACSCalibur (BD Biosciences).

\section{Functional assay for hepatocytes}

To test hepatic functions with stem cell iHeps at the final day of stage IV (day 20 of differentiation), the differentiated cells were dissociated in Accutase for $30 \mathrm{~min}$ and seeded on Collagen I-coated plates with HMM medium. After overnight incubation at $37^{\circ} \mathrm{C}$, each functional test such as periodic acid Schiff (PAS) staining, low-density lipoprotein (LDL) uptake, albumin/urea secretion, CYP450 enzyme activity and drug metabolism was carried out as described in the following. HepG2 and $\mathrm{p}$-Heps were used as control cells.

\section{PAS staining}

To detect glycogen storage, PAS staining was performed at day 21 of differentiation. The cells were fixed for $5 \mathrm{~min}$ with 4\% PFA and oxidized for $5 \mathrm{~min}$ in periodic acid (Sigma-Aldrich, MO, USA) at room temperature. After rinsing with distilled water for $5 \mathrm{~min}$, the cells were treated with Schiff's reagent (Sigma-Aldrich) for $15 \mathrm{~min}$.

\section{LDL uptake}

To estimate uptake of low LDL, LDL-DyLight ${ }^{\mathrm{Tm}} 550$ (Abcam) was diluted with culture medium (1:100). Cells were incubated with LDL-DyLight ${ }^{\mathrm{m}} 550$ working solution for $4 \mathrm{~h}$ at $37{ }^{\circ} \mathrm{C}$. The medium was replaced with fresh culture medium. Immunofluorescence was detected under a fluorescence microscope (Axiovert).

\section{Albumin and urea secretion}

The culture medium was changed at day 21 of differentiation, and the cells were additionally incubated for $24 \mathrm{~h}$ at $37{ }^{\circ} \mathrm{C}$. The supernatants collected from each well were centrifuged for $5 \mathrm{~min}$ at $3000 \times g$ to remove floating cells and stored at $-20{ }^{\circ} \mathrm{C}$ until assay. The albumin and urea amounts in culture medium were measured using an $\mathrm{Al}-$ bumin Human ELISA kit (Abnova, CA, USA) and urea assay kit (Cell Biolabs, CA, USA), respectively, according to the manufacturer's instructions. Absorbance was read on a luminometer (FlexStation III) at a wavelength of $450 \mathrm{~nm}$ for albumin and $630 \mathrm{~nm}$ for urea. The albumin and urea amounts were calculated using each standard curve and normalized by protein concentration $(\mathrm{mg} / \mathrm{ml})$.

\section{CYP450 enzyme activity}

CYP1A2 and CYP3A4 enzyme activities were measured using the CYP450-Glo ${ }^{\mathrm{mm}}$ assay kit (Promega, WI, USA) according to the manufacturer's instructions. The supernatants were removed, and the cells were incubated with substrate (Luciferin-1A2 for CYP1A2 and Luciferin-IPA for CYP3A4) for $1 \mathrm{~h}$. The supernatants of each well were transferred to white opaque 96-well plates. CYP450 activities were then measured using a luminometer (FlexStation III). The results were expressed as a relative activity for control. 


\section{Drug clearance}

To evaluate drug metabolism, $1 \mu \mathrm{M}$ aflatoxin B1 (Sigma-Aldrich) and $100 \mu \mathrm{M}$ acetaminophen (Sigma-Aldrich) diluted with HMM medium treated QIA7-iHeps for $24 \mathrm{~h}$, and medium containing test drugs was used as control (no cells). The supernatants were collected, and the concentrations of each compound in the supernatants were determined by HPLC (Waters 2996; Waters, MA, USA). Drug clearance in p-Heps was also performed under the same method. The values were normalized by protein concentration $(\mathrm{mg} / \mathrm{ml})$ and expressed by the percentage of control.

\section{Teratoma formation}

For in-vivo cell transplantation, QIA7 and QIA7-iHeps (day 7 of differentiation) pretreated with and without YM155 (5 nM) were dissociated by Dispase and Accutase, respectively. Approximately $1 \times 10^{6}$ cells were prepared in DMEM/F12 $(50 \mu \mathrm{l})$ and mixed with Matrigel (1:1) on ice. The mixture was injected into the testis of 6-week-old nude mice (BkINbt:BALB/c/nu/nu; NARABiotech, Republic of Korea). Six or seven weeks later, the teratomas were dissected. Tumor masses were fixed with $10 \%$ neutral buffered formalin (Sigma-Aldrich). Paraffinembedded tissues were sectioned and stained with hematoxylin and eosin (H\&E) and analyzed in the Cell Imaging-Histology core facility at the Quarantine \& Inspection Agency.

\section{Statistical analysis}

Results were expressed as the mean \pm standard deviation (SD) for triplicate experiments $(n=3)$. The statistical significance was determined using Statistica5.5 (StatSoft, OK, USA) with one-way analysis of variance (ANOVA) and post-hoc comparisons between the control group and each treatment group using Duncan's multiple comparison test. $p<0.05$ was considered statistically significant.

\section{Results}

\section{Hepatic differentiation of human PSCs with the modified} protocol

To achieve hepatic differentiation, human PSCs were differentiated to hepatocytes for 20 days via four sequential stages; definitive endoderm (5 days), hepatic endoderm (5 days), hepatic specification (5 days) and hepatic maturation (5 days) (Fig. 1a). Human PSCs were induced to definitive endoderm using Activin A $(100 \mathrm{ng} / \mathrm{ml})$ for 5 days. During the whole induction period of definitive endoderm, a large number of differentiated cells was easily detached from the plate. Thus, the relevant cell number was needed for protocol optimization. In our preliminary results, the optimal density of cells was approximately $2 \times$ $10^{4}-3 \times 10^{4}$ cells $/ \mathrm{cm}^{2}$ in human PSCs (data not shown).
Under this condition, QIA7 showed stage-specific morphology at each stage (Fig. 1b). For expression of stagespecific marker genes shown in Fig. 1c, pluripotent genes such as NANOG and OCT4 rapidly decreased at stage I. On the contrary, GATA4, SOX17, FOXA2 and CXCR4 as definitive endodermal markers were highly expressed at stage I, and expression diminished thereafter. For the hepatic markers, expression of HNF4A and AFP started to be increased at stage II, and expression was maximized at stage III. $A L B$ was not detected at stage II, and expression was sharply enhanced at stage III. However, three hepatic marker genes were decreased at stage IV. The expression patterns of genes were similar to those of marker proteins (Fig. 1d and Additional file 2: Figure S1). Cytokeratin 18 (CK18)-positive cells were detected during the whole period of differentiation. QIA7-iHeps showed a typical hepatocyte phenotype and glycogen storage at the final stage (Fig. 1e).

\section{Characterization of nonhepatic lineage cells derived from USCs}

To characterize the residual USCs, pluripotent genes and marker proteins were investigated under our modified protocol of hepatic differentiation. The expression of NANOG and OCT4 did not disappear until day 15 of differentiation (stage III), although the expression sharply declined by day 2 of differentiation (Fig. 2a). Most of the clusters still remaining at stage I (day 5 of differentiation) expressed pluripotent markers such as NANOG, OCT4, TRA-1-60 and SSEA-4 (Fig. 2b). Thus, we hypothesized that the USCs caused cellular heterogeneity in the hepatic differentiation. QIA7 actively differentiated to definitive endodermal cells after exposure to Activin A at stage I. However, many cell clusters still expressing the pluripotent markers were observed on the surrounding edges of definitive endodermal cells at stage I (Fig. 2c, a'). The clusters were tightly aggregated as soon as BMP2 and FGF4 were treated at stage II (Fig. 2c, b'). Thereafter, the cell population derived from the aggregated cells rapidly expanded at stage III (Fig. 2c, c'). This cell population (Fig. 2c, d', magnified in area 1 (A1) of Fig. 2c, c') accounted for the majority of the cell population at the final stage of differentiation and distinguishable from typical hepatic phenotype in area 2 (A2) of Fig. 2c, c' (Fig. 2c, e'). During immunostaining, the cells of A1 were negative for ALB, but most of the cells in A2 were positive (Fig. 2c, f'). To further characterize $\mathrm{A} 1$ and $\mathrm{A} 2$, each cell population was separately collected according to distinct cell morphology. Interestingly, expression of NANOG and OCT4 was still present in A1 whereas it was not detected in A2 (Fig. 2d). As compared with A1, A2 highly expressed endodermal marker genes (AFP, $A L B, S O X 17$ and FOXA2) (Fig. 2e). 


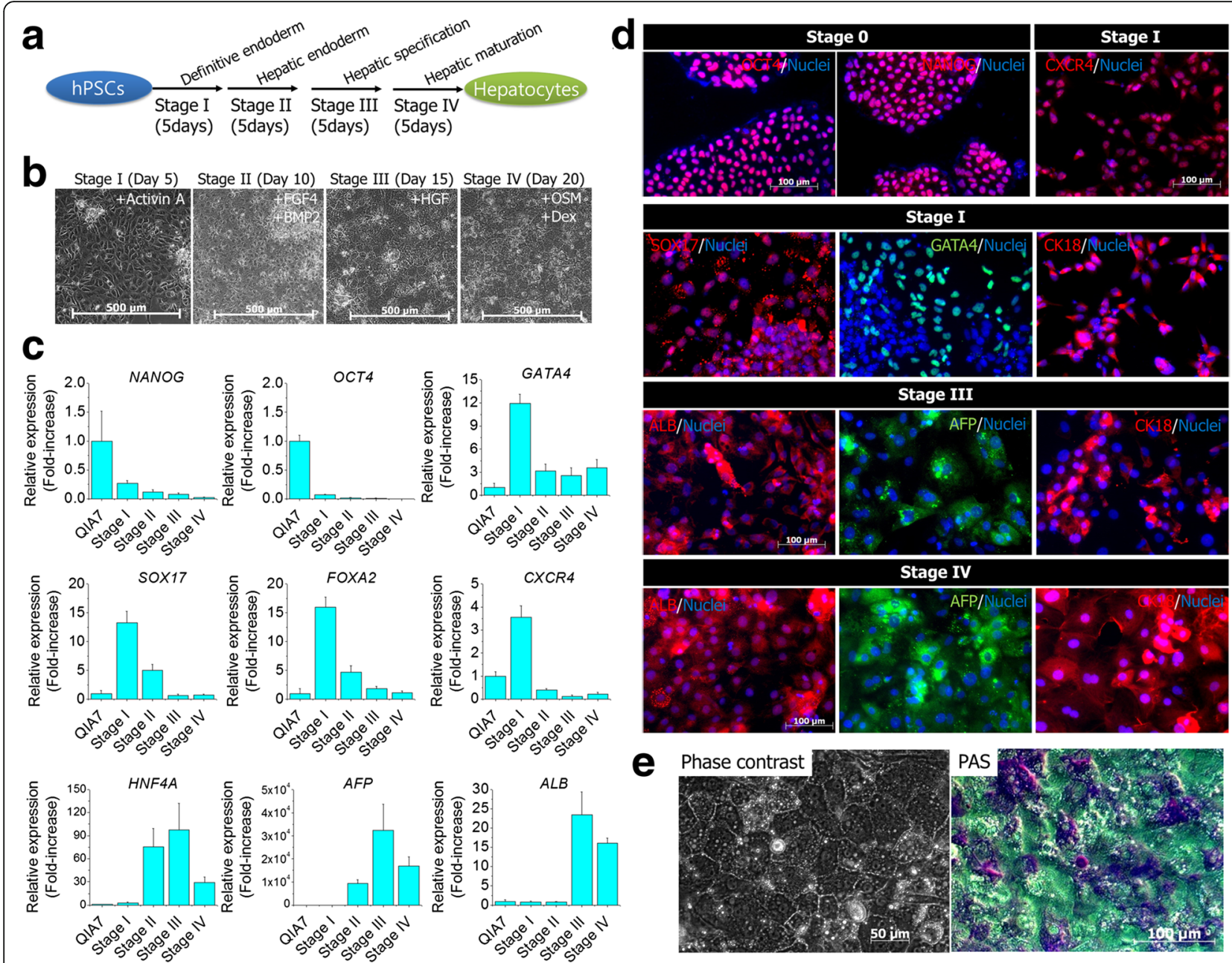

Fig. 1 Hepatic differentiation of human PSCs. The modified protocol of hepatic differentiation was distinct at four stages; definitive endoderm, hepatic endoderm, hepatic specification and hepatic maturation (a). Under this sequential induction condition, the development of QIA7 to hepatocytes was confirmed at each stage in the aspects of morphological changes (b) and expression of stage-specific marker genes (c) and proteins (d). The differentiated hepatocytes showed a typical hepatocyte-like shapes and glycogen synthesis at stage IV (e). hPSC human pluripotent stem cell, AFP alphafetoprotein, ALB albumin, BMP2 bone morphogenetic protein 2, CK18 cytokeratin 18, CXCR4 C-X-C chemokine receptor type 4, FGF4 fibroblast growth factor 4, FOXA2 forkhead box protein A2, GATA4, transcription factor GATA-4, HGF hepatocyte growth factor, HNF4A hepatocyte nuclear factor 4 alpha, OCT4 octamer-binding transcription factor, OSM oncostatin M, PAS periodic acid Schiff, SOX17 sex determining region Y-Box 17

\section{Effects of YM155 on hepatic differentiation}

We hypothesized that highly qualified and quantified hepatocytes could be obtained if USCs are effectively removed during hepatic differentiation. To perform selective removal of USCs, YM155 was used in this study. Firstly, serial concentrations of YM155 were used to treat human PSCs (QIA7 and WA01), differentiating cells (QIA7-iHeps) and hAT-SCs as a somatic cell for determining the relevant treatment concentration (Fig. 3a). Each cell type showed different dose responses against YM155 (Fig. 3a). The inhibitory concentration $50\left(\mathrm{IC}_{50}\right)$ of QIA7, WA01, QIA7-iHeps and hAT-SCs was $8 \mathrm{nM}, 14 \mathrm{nM}, 130 \mathrm{nM}$ and $3086 \mathrm{nM}$, respectively. Human PSCs were affected by lower concentrations of YM155 than hAT-SCs and QIA-iHeps.
Therefore, we selected the ranges of YM155 (1-100 $\mathrm{nM})$ for later study. To determine an optimal time point of YM155 treatment, the selected concentrations of YM155 were treated at stage I and stage II. In our preliminary study, the more YM155 treatment at stage I, the more cells survived (Additional file 2: Figure S2). Most of the differentiating cells were easily detached from plates by YM155 treatment at stage I. Therefore, YM155 was not applicable at stage I. On the contrary, the differentiating cells were more tolerant to YM155 treatment at stage II (day 6 of differentiation) than at stage I (day 4 of differentiation) (Fig. 3b). To investigate cytotoxic effects of YM155 at stage II, we estimated changes of apoptosis-related genes on the whole differentiation (Additional file 2: Figure S3). BIRC5 


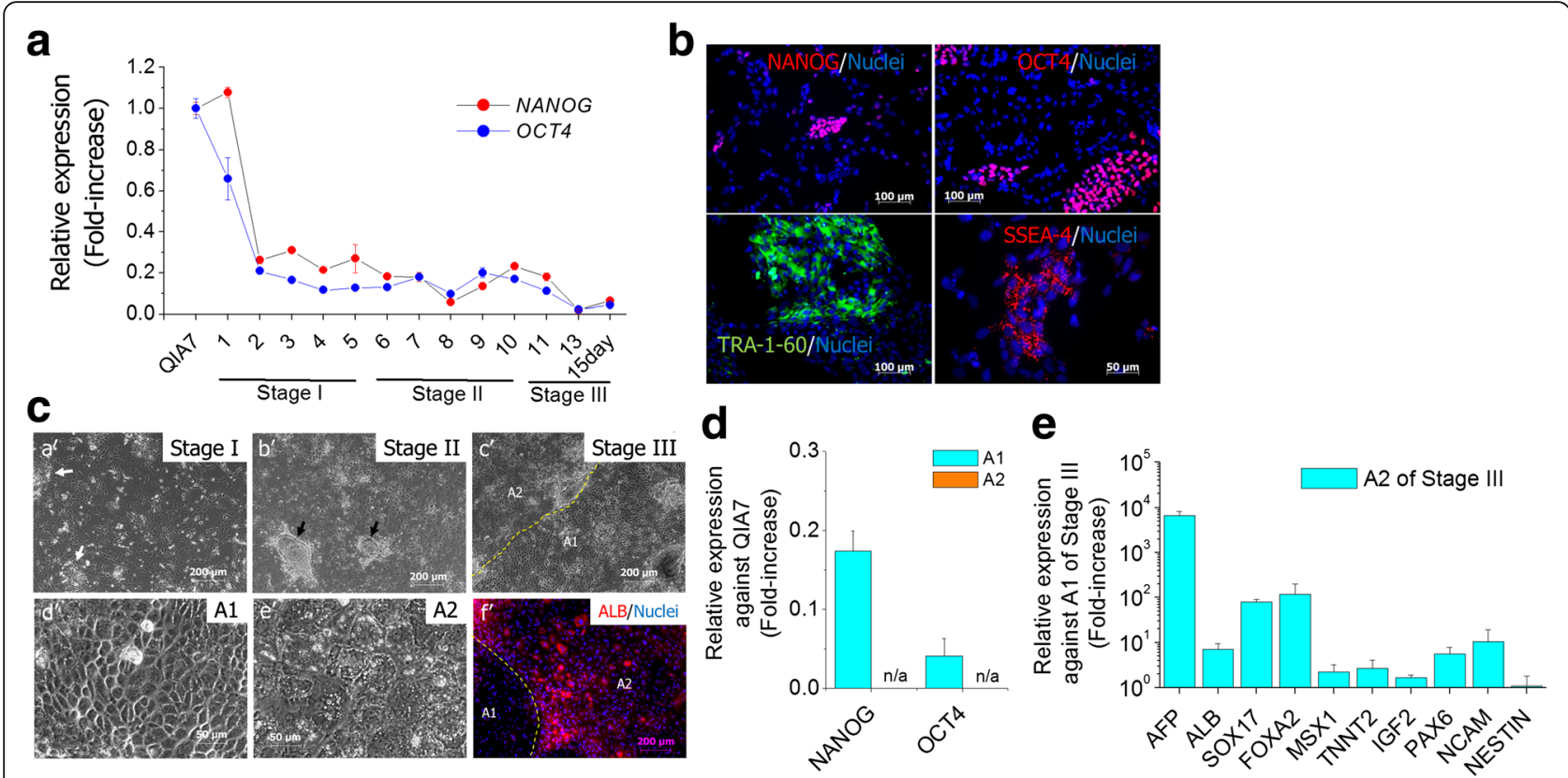

Fig. 2 Characterization of nonhepatic lineage cells derived from USCs during hepatic differentiation. Expression of NANOG and OCT4 as pluripotent genes was traced to confirm the presence of residual USCs during hepatic differentiation (a). Additionally, human pluripotent stem cell-specific markers such as NANOG, OCT4, TRA-1-60 and SSEA-4 were detected in cell clusters at day 5 of hepatic differentiation (stage I) (b). (c) The cell clusters (arrow) of stage I ( $a^{\prime}$ ) were tightly aggregated during stage II (arrow) ( $\left.b^{\prime}\right)$. Thereafter, the aggregated cells rapidly expanded to nonhepatic lineage cells (area 1 (A1)) distinguishable from typical hepatic phenotype cells (area 2 (A2)) at stage III (c'). (d', $\left.e^{\prime}\right)$ Magnification of $A 1$ and $A 2$ in $\left(C^{\prime}\right)$, respectively. $A 1$ and $A 2$ were stained with Albumin antibody $(f)$. Nuclei were stained with Hoechst33258. For further characterization, expression of pluripotent genes (NANOG and OCT4) (d) and lineage-specific genes (AFP, ALB, SOX17, MSX1, TNNT2, IGF2, PAX6, NCAM and NESTIN) (e) was compared between A1 and A2 at stage III. AFP alpha-fetoprotein, ALB albumin, FOXA2 forkhead box protein A2, IGF insulin growth factor2, MSX1 msh homeobox-1, NCAM neural cell adhesion molecule, OCT4 octamer-binding transcription factor, PAX6 paired box protein Pax-6, SOX17 sex determining region Y-Box 17, TNNT2 cardiac muscle troponin T

was down-regulated from day 1 of differentiation and maintained during the whole hepatic differentiation. $B A X$, a pro-apoptotic gene, was up-regulated from stage II to stage III. The expression of $B C L-2$, an antiapoptotic gene, was not detectable or was at a very low level (data not shown). After YM155 treatment at stage II (day 6 of differentiation), BIRC5 and BCL-2 were dose-dependently decreased as compared with DMSO whereas $B A X$ was increased (Fig. 3c). Likewise, Caspase-3 activity was significantly increased by a lower concentration of YM155 in QIA7 more than QIA7-iHeps but was not affected in hAT-SCs (Fig. 3d). These results were very similar with Annexin V staining (Additional file 2: Figure S4). In the change of cell phenotype, cell clusters were effectively removed by more than $5 \mathrm{nM}$ of YM155 but not by $1 \mathrm{nM}$ and DMSO (Fig. 3e). However, all cells were dead with $100 \mathrm{nM}$ of YM155 treatment. The cells affected by YM155 were closely consistent with TRA-1-60 $0^{+}$cells (Additional file 2: Figure S5). Likewise, $\mathrm{OCT}^{+}$cells were significantly decreased by more than $5 \mathrm{nM}$ of YM155 in FACS analysis (Fig. 3f). In the genetic analysis, expression of NANOG and OCT4 was decreased and expression of endodermal marker genes such as CXCR4, SOX17 and FOXA2 was increased in a dose-dependent manner (Fig. 3g). Taken together, the residual USCs were effectively eliminated by YM155 at stage II (day 6 of differentiation) as compared with differentiating cells and somatic cells.

\section{Evaluation of hepatic induction followingYM155 treatment at stage II}

The level of hepatic differentiation was evaluated at stage III (day 15 of differentiation) after YM155 treatment for $24 \mathrm{~h}$ at stage II (day 6 of differentiation). Cellular homogeneity of differentiated cells was observed by $5 \mathrm{nM}$ of YM155 compared with DMSO and $1 \mathrm{nM}$ of YM155 (Fig. 4a). In the group treated with $5 \mathrm{nM}$ of YM155, expression of hepatic endodermal genes $A L B$, $A F P$ and HNF4A increased significantly in the differentiated cells compared with DMSO. On the contrary, expression of PAX6, NESTIN and NCAM for ectodermal markers and MSX1 and TNNT2 for mesodermal genes was decreased in the cells (Fig. 4b). To estimate the purity of hepatocytes in each population, FACS analysis was conducted by immunostaining ALB antibody. Albumin-positive cells increased approximately 1.8 -fold 


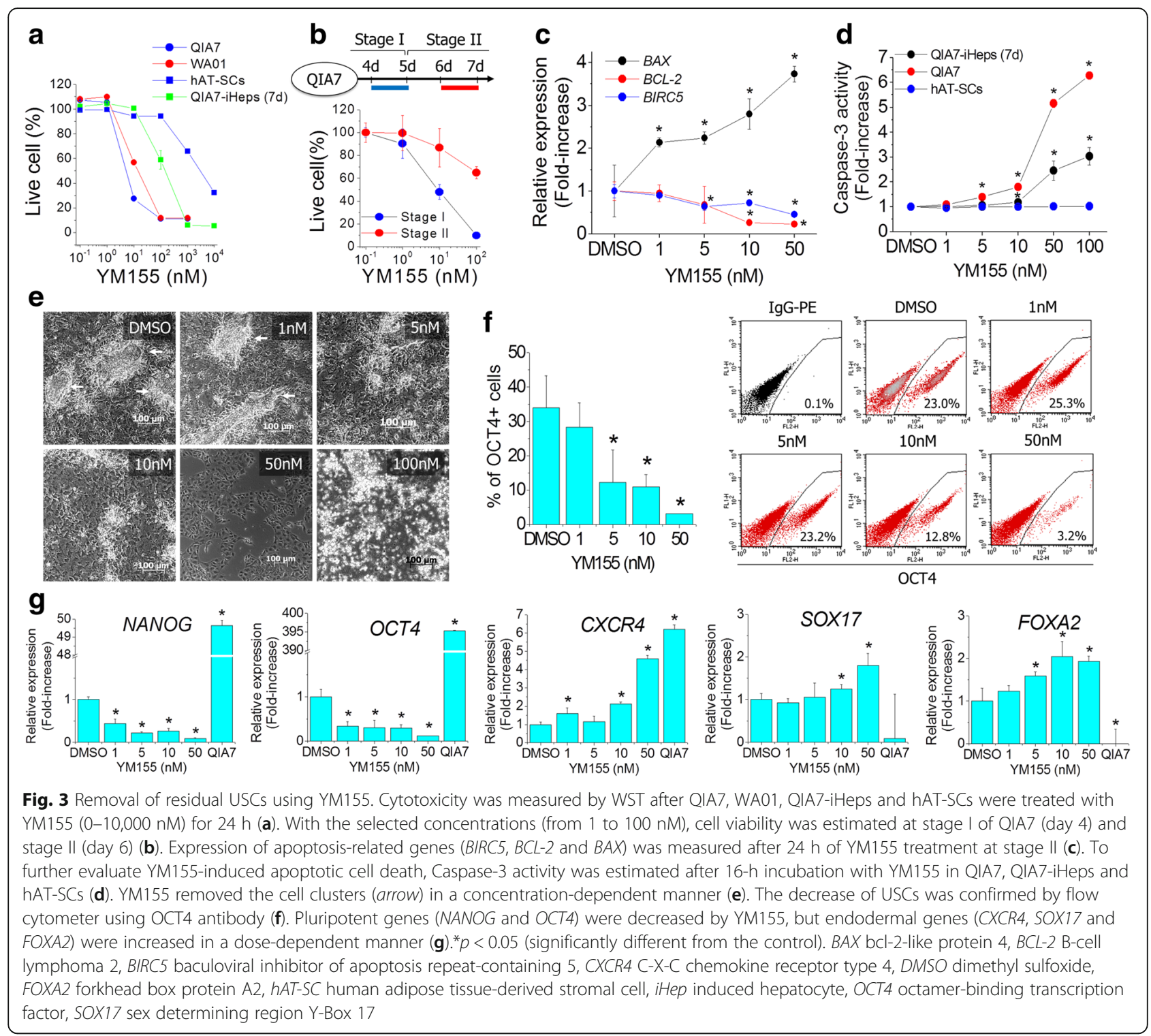

with $5 \mathrm{nM}$ of YM155 (75 $\pm 6 \%)$ and 1.6-fold with 10 $n M(69 \pm 2 \%)$ compared with DMSO (42 $\pm 6 \%)$ (Fig. 4c). In a further functional assay conducted on QIA7-iHeps pretreated with YM155 at stage II, activities of CYP450 enzymes showed significant increases in $5 \mathrm{nM}$ by approximately 2.1-fold for CYP1A2 and 2.8-fold for CYP3A4 (Fig. 4d). In immunocytochemical staining, however, QIA7-iHeps showed weak immunoreactivity against CYP1A2 but strong immunoreactivity for CYP3A4 (Fig. 4e).

Collectively, $5 \mathrm{nM}$ of YM155 at stage II was chosen as the optimal concentration and time point to generate QIA7-iHeps in an aspect of cell purity and function. With $5 \mathrm{nM}$ of YM155 at stage II, WA01-iHeps differentiated from hESCs showed typical characteristics of hepatocytes, cuboidal morphology and glycogen synthesis
(PAS staining), LDL uptake and immunoreactivity against AFP and ALB (Fig. 5a). Further validation was conducted with $5 \mathrm{nM}$ of YM155 in QIA7-iHeps and WA01-iHeps and compared with control cells (HepG2 and p-Heps). The amounts of albumin increased over approximately 2.2-fold in QIA7-iHeps and 1.3-fold in WA01-iHeps (Fig. 5b). In urea secretion, the increases showed 1.3-fold in QIA7-iHeps and no significant difference in WA01-iHeps (Fig. 5c). Even though the tested hepatic functions were improved by YM155 treatment $(5 \mathrm{nM})$ and showed a similar level with $\mathrm{p}$ Heps, the clearance ratios of tested compounds in QIA7-iHeps and WA01-iHeps were significantly lower than those of p-Heps; aflatoxin B1 $(25.9 \%, 26.4 \%$ and $66.4 \%$, respectively) and acetaminophen $(17 \%, 12.6 \%$ and $24.9 \%$, respectively) (Fig. $5 \mathrm{~d}$ ). 

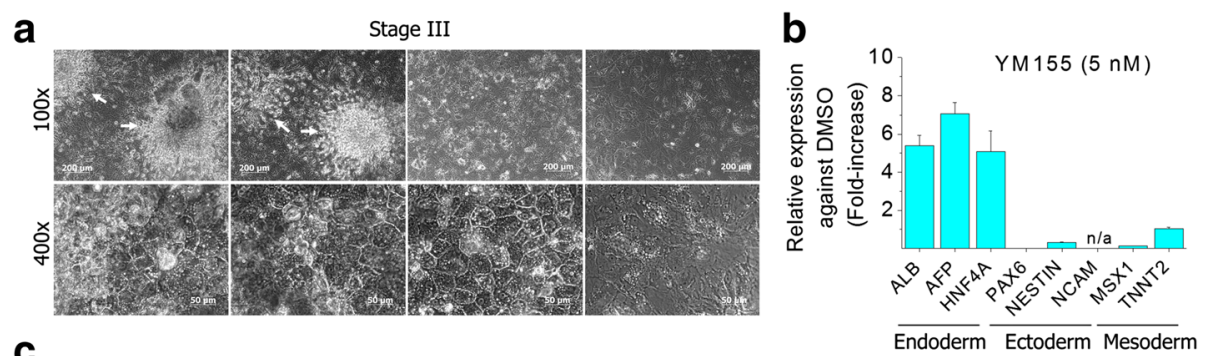

C
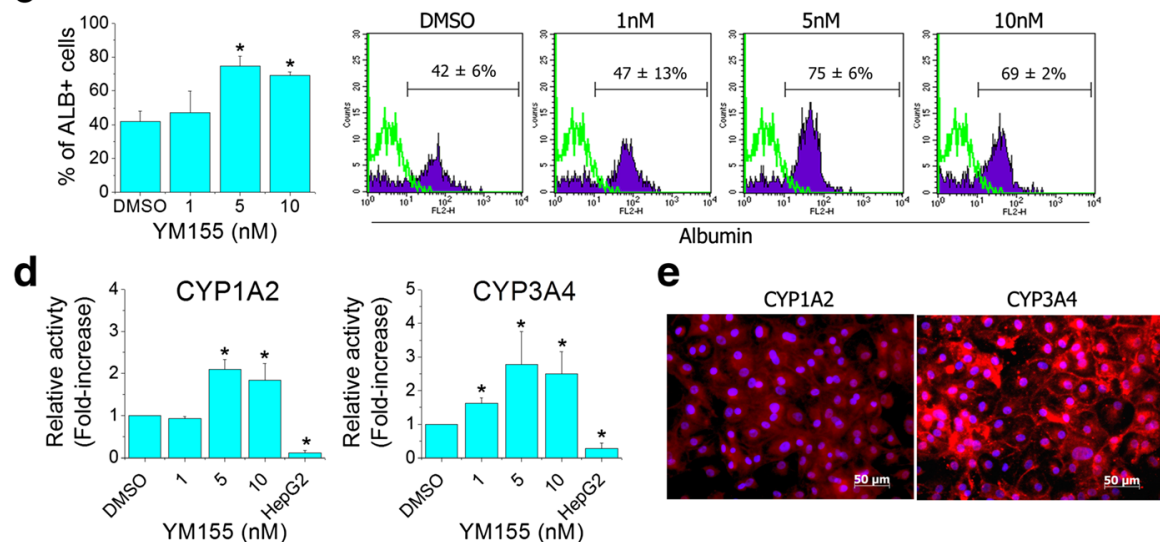

Fig. 4 Characterization of hepatic induction following YM155 treatment at stage II. At stage III, the homogeneity of the cell population was observed in 5 and $10 \mathrm{nM}$ YM155 treatment of stage II (day 6), but nonhepatocyte-like cells (arrow) derived from USCs still appeared in $1 \mathrm{nM}$ YM155 like DMSO (a). Expression of hepatic endoderm (AFP, ALB and HNF4A), ectoderm (PAX6, NESTIN and NCAM) and mesoderm (MSX1 and TNNT2) marker genes was estimated at 15 days of differentiation (b). At stage IV, albumin-positive cells were measured by flow cytometer $(n=3)$ (c) and enzyme activities of CYP1A2 and CYP3A4 were also estimated with QIA7-iHeps (d). QIA7-iHeps showed weak immunoreactivity against CYP1A2 but strong against CYP3A4 (e). ${ }^{*} p<0.05$ (significantly different from the control). AFP alpha-fetoprotein, ALB albumin, DMSO dimethyl sulfoxide, HNF4A hepatocyte nuclear factor 4 alpha, PAX6 paired box protein Pax-6, MSX1 Msh homeobox-1, NCAM neural cell adhesion molecule, TNNT2 cardiac muscle troponin T

\section{YM155 induces selective cell death of USCs and prevents teratoma formation}

Based on our in-vitro results, we tested whether YM155 treatment could prevent teratoma formation in vivo after cell transplantation. Firstly, we confirmed that expression of pluripotent marker genes, such as NANOG and OCT4, was rapidly decreased after $5 \mathrm{nM}$ of YM155 treatment compared with control, and the two genes completely disappeared at day 15 of differentiation (Fig. 6a). For cell transplantation, we prepared QIA7 and QIA7-iHeps (day 7 of differentiation) pretreated with $\left(\mathrm{YM} 155^{+}\right)$or without $5 \mathrm{nM}$ of YM155 (YM155 ${ }^{-}$) for $24 \mathrm{~h}$, respectively (Fig. 6b). As shown in Fig. 6c, d, all mouse testes $(n=5)$ injected with QIA7 (YM155 $\left.{ }^{-}\right)$developed teratomas after 6-7 weeks whereas the counterparts

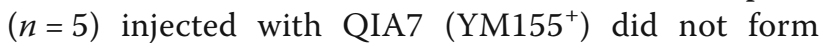
any teratoma-like tumor mass. Interestingly, we observed that QIA7-iHeps (YM155 ) could develop tumor mass (two of five testes; 40\%) but not in YM155 pretreatment $\left(\mathrm{YM} 155^{+}\right)$. In the histological analysis, these teratomas showed tissues of all three germlayers (Fig. 6e and Additional file 2: Figure S6).

\section{Discussion}

In the present study, we confirmed that human PSCs differentiated into hepatocyte-like cells via four sequential steps modified from the protocol of Cai et al. [1] by demonstrating the expression of hepatic genes, such as $H N F 4 A, A F P$ and $A L B$, and functional hepatic proteins, such as ALB and AFP. Contrary to our expectation, expression of $A L B$ and $H N F 4 A$ decreased at stage IV. This might be derived from a nonoptimal culture condition of stage IV (no evaluation in this study) or a relatively low ratio of hepatic purity due to expansion of nonhepatic lineage cells. Morphologically nonhepatic cells (A1) were actually observed at stage III and expanded rapidly during the differentiation. This cell population (A1) showed a distinct morphology against hepatic population as a counterpart (A2) and also contained USCs (Fig. 2d). Additionally, those cells were negative for albumin protein (mature hepatocyte marker), overexpressed AFP and IGF2 (fetal hepatic genes) and expressed mesoderm and ectoderm origin genes. These findings suggest that these cell clusters were derived from USCs and finally reduced cellular homogeneity at the final stage of hepatic differentiation. Therefore, the efficiency of hepatic 


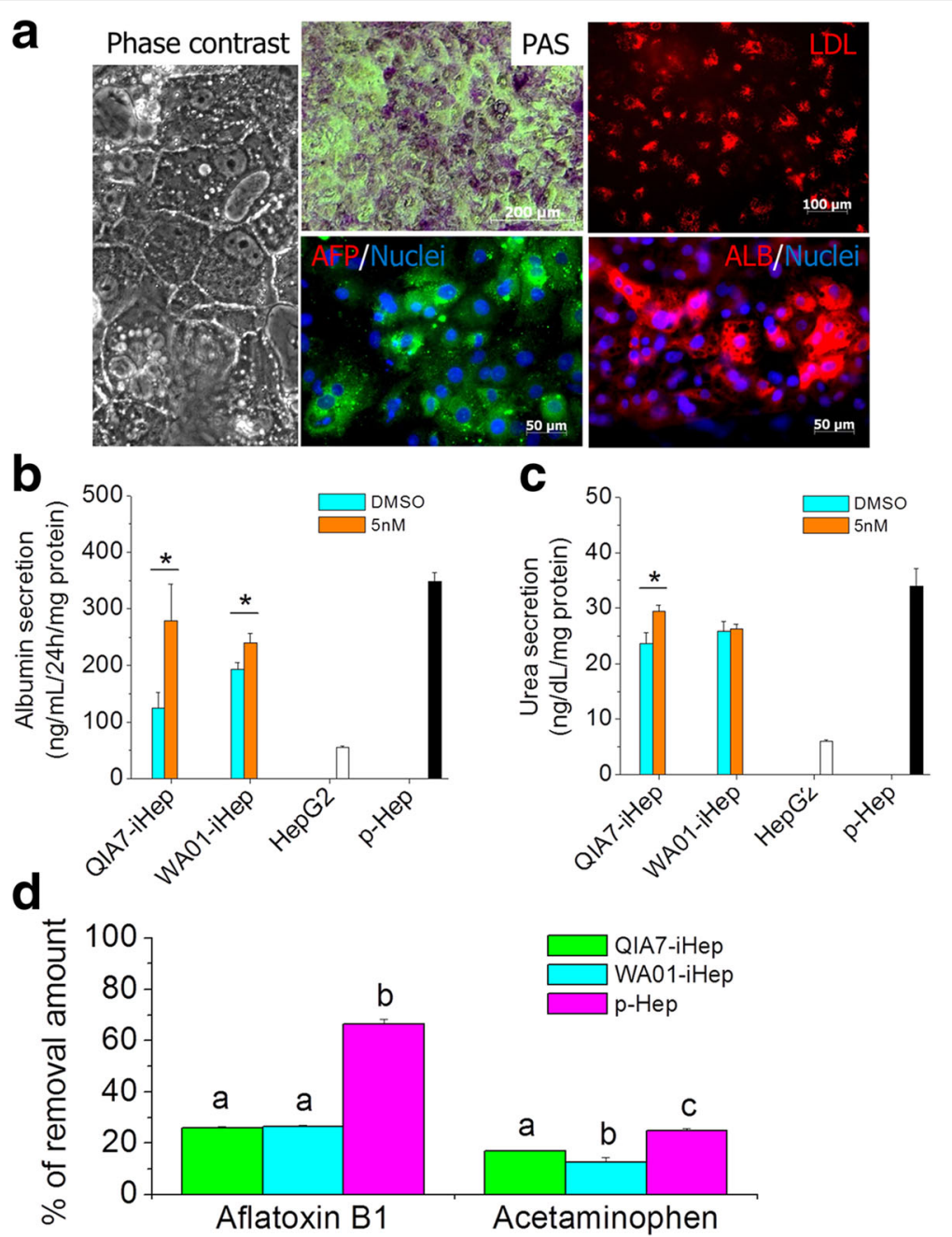

Fig. 5 Hepatic functional tests with stem cell iHeps pretreated withYM155 at stage II. WA01-iHeps pretreated with 5 nM YM155 at stage II showed the typical hepatic morphology, glycogen synthesis, LDL uptake and immunoreactivity of AFP and ALB (a). With stem cell iHeps (QIA7-iHeps and WA01-iHeps) pretreated by $5 \mathrm{nM}$ YM155 selected as the optimal concentration at stage II, the amount of albumin (b) and urea (c) secreted for $24 \mathrm{~h}$ at day 22 of differentiation was measured and compared with DMSO control, HepG2 and p-Heps. Also, drug clearance was determined with aflatoxin B1 $(1 \mu \mathrm{M})$ and acetaminophen $(100 \mu \mathrm{M})(\mathbf{d})$. After each compound was incubated with QIA7-iHeps, WA01-iHeps and p-Heps for 24 h, the concentration of each compound was measured using the HPLC method (d). ${ }^{*} p<0.05$ and different superscript letters indicate significant differences. AFP alpha-fetoprotein, ALB albumin, DMSO dimethyl sulfoxide, iHep induced hepatocyte, LDL low density lipoprotein, PAS periodic acid Schiff

differentiation could be improved when USCs are selectively eliminated during differentiation.

Small molecules target anti-apoptotic factors to remove residual USCs $[17,18,22]$. Of these small molecules, YM155 in particular triggers apoptosis of USCs but not that of their differentiated derivatives [18]. Likewise, we also demonstrated that human PSCs were uniquely affected by YM155 within the specific ranges (1-10 nM) more than differentiating cells and somatic cells (Fig. 3a). However, it was difficult to apply YM155 at stage I because almost all cells were easily detached from the plate. Therefore, YM155 was applied at stage II. After YM155 treatment, we confirmed apoptotic cell death by apoptotic gene expression, Caspase-3 activity and Annexin V staining. This apoptosis was defined in USCs positive against TRA-1-60 and OCT4. Taken together, YM155 effectively removed USCs at stage II of the differentiation procedure. Finally, this selective elimination resulted in enhancing the expression of endodermal marker genes (CXCR4, SOX17 and FOXA2) at stage II and hepatic marker genes (ALB, AFP and HNF4A). We think these increases might be compensation following the decrease of nonspecific lineages.

Drug metabolization is one of the key functions of the liver. The role is primarily conducted by phase I enzymes (CYP450 enzymes) during xenobiotic exposure. 


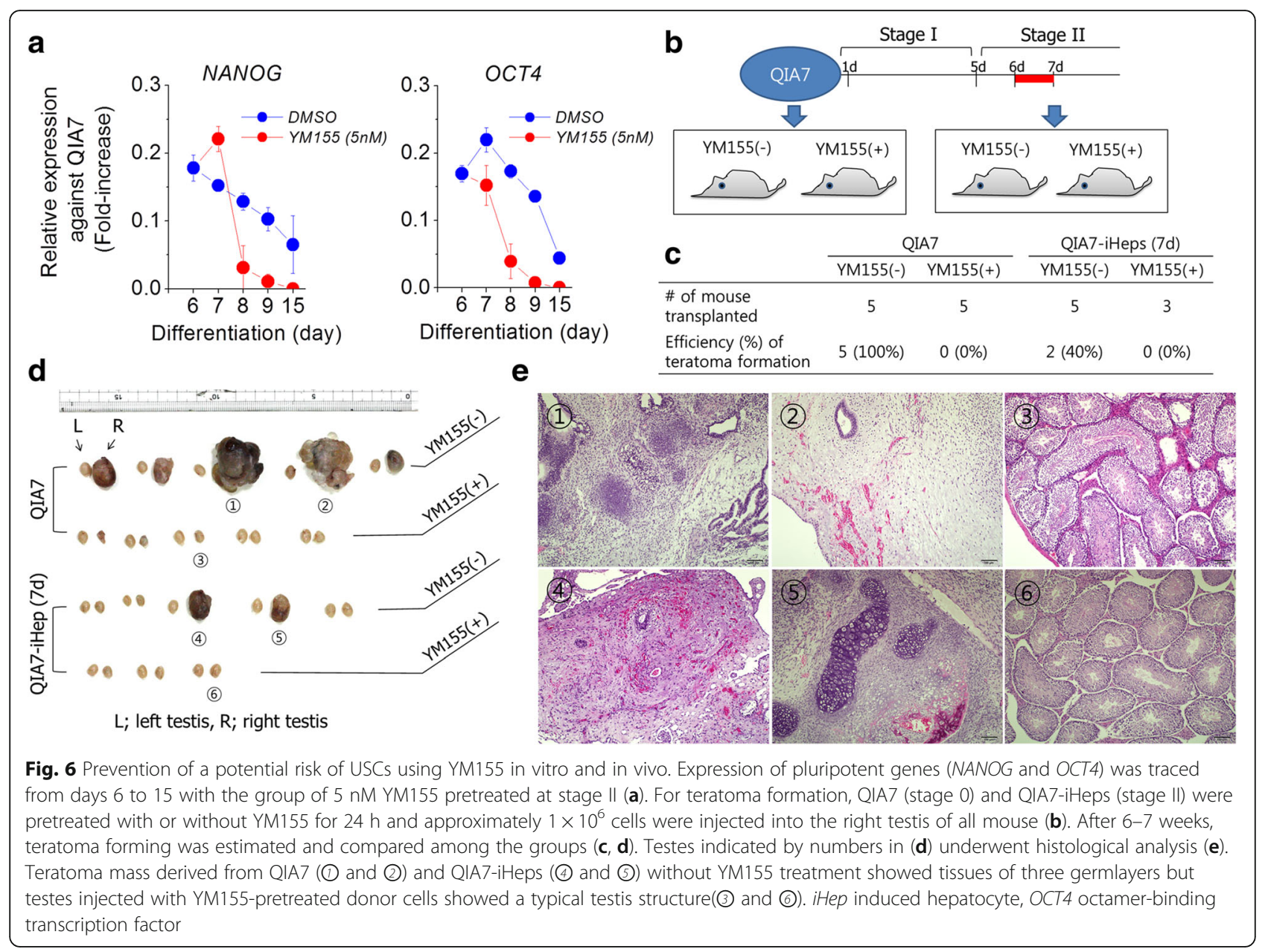

Here, the activity of CYP1A2 and CYP3A4 in QIA7iHeps was significantly increased by $5 \mathrm{nM}$ of YM155 compared with no treatment due to the decrease of the nonhepatic population. However, drug clearance of QIA7-iHeps and WA01-iHeps was significantly lower than that of p-Heps. Aflatoxin B1 and acetaminophen used in this study were metabolized mainly by CYP1A2 and CYP3A4 in hepatocytes. In our previous study, the activity of CYP1A2 and CYP3A4 in QIA7-iHeps and WA01-iHeps was approximately half that of primary hepatocytes [20]. The data are considerably correlated with drug clearance in this study. These lower metabolic activities in QIA7-iHeps and WA01-iHeps compared with $\mathrm{p}$-Heps might be derived from the individual variation of CYP450 genes (i.e., single nucleotide polymorphism) and/or levels of hepatic purity and maturation. A combination of YM155 and additional factors [5, 7, 23, 24] as well as a $3 \mathrm{D}$ culture system $[8,25,26]$ will be able to demonstrate these issues.

We confirmed that pluripotent genes (NANOG and OCT4) were completely down-regulated after YM155 treatment and were nearly prevented at the final day of stage II. Finally, expression was not detected at 15 day of differentiation. Under our optimized concentration of YM155 at stage II, USCs could efficiently induce apoptotic cell death. Also, YM155 effectively prevented teratoma formation in our invivo study. Interestingly, teratoma occurrence was observed in the transplantation of QIA7-iHeps without YM155. This might be derived from residual USCs on differentiation. Residual USCs accounted for approximately $20-30 \%$ of the whole differentiating cells based on the expression of pluripotent marker genes and proteins at stage II. Arithmetically, we can presume that approximately $2 \times 10^{5}$ cells remaining as USCs were injected into the testis. Finally, these cells induced teratoma (40\%). Based on a previous study [27], this cell number is sufficient to induce teratoma formation. On the contrary, QIA7-iHeps pretreated with YM155 did not form anything. Regarding cell therapy, the elimination of residual USCs is one of the major concerns to guarantee safety. Based on our evidence, we will be able to utilize the differentiated cells safely as a donor in cell transplantation. 


\section{Conclusions}

YM155 could selectively eliminate USCs with an effective treatment time point and optimal concentration on hepatic differentiation. The removal of residual USCs enhanced the efficiency of hepatic differentiation as well as reduced a potential risk of teratoma formation. To our knowledge, this is the first report to demonstrate the effects of YM155 on in-vitro hepatic differentiation. Based on recently advanced technology of hepatic differentiation, application of YM155 will allow generation of a pure hepatocyte population and safety of cell transplantation in regenerative medicine.

\section{Additional files}

Additional file 1: is Table S1. presenting a list of primer sets and Table S2. presenting antibody information. (DOCX $31 \mathrm{~kb}$ )

Additional file 2: contains supplementary materials and methods presenting TRA-1-60 livestaining and Annexin V staining; Figure S1. showing the immunocytochemical phenotype stage on hepatic differentiation; Figure S2. showing the YM155 effect on stage I; Figure S3. showing expression of apoptosis-related genes (BIRC5 and BAX) during the whole hepatic differentiation of QIA7; Figure S4. showing Annexin V staining with QIA7, QIA7-iHeps (7 days of differentiation) and hAT-SCs after YM155 treatment for 24 h; Figure S5. showing the livestaining of TRA-1-60 at stage II (day 7 of differentiation) after YM155 treatment for $24 \mathrm{~h}$; and Figure S6. showing the histological analysis of teratomas derived from QIA7 and QIA7-iHeps without pretreatment of YM155. (DOCX $11505 \mathrm{~kb})$

\section{Abbreviations}

AFP: Alpha-fetoprotein; ALB: Albumin; BAX: BCl-2-like protein 4; BCL-2: B-cell lymphoma 2; BIRC5: Baculoviral inhibitor of apoptosis repeat-containing 5; BMP2: Bone morphogenetic protein2; CK18: Cytokeratin 18; CXCR4: C-X-C chemokine receptor type 4; CYP450: Cytochrome P450; DMEM: Dulbecco's Modified Eagle Medium; DMSO: Dimethyl sulfoxide;

EDTA: Ethylenediaminetetraacetic acid; EMEM: Eagle's minimum essential medium; FBS: Fetal bovine serum; FGF4: Fibroblast growth factor 4; FOXA2: Forkhead box protein A2; GAPDH: Glyceraldehyde 3-phosphate dehydrogenase; GATA4: Transcription factor GATA-4; HGF: Hepatocyte growth factor; HNF4A: Hepatocyte nuclear factor 4 alpha; IGF2: Insulin growth factor2; MSX1: Msh homeobox-1; NCAM: Neural cell adhesion molecule; OCT4: Octamer-binding transcription factor; OSM: Oncostatin M; PAS: Periodic acid Schiff; PAX6: Paired box protein Pax-6; ROCK: Rhoassociated protein kinase; SOX17: Sex determining region Y-Box 17; TNNT2: Cardiac muscle troponin T

\section{Acknowledgements}

\section{Not applicable.}

Language service; https://webshop.elsevier.com/languageservices (LE191504).

\section{Funding}

This project was supported by research funds from Animal and Plant Quarantine Agency (QIA), Republic of Korea.

\section{Availability of supporting data}

All data generated or analyzed during this study are included in this published article and its supplementary information files.

\section{Authors' contributions}

S-JK, H-GK, H-OK and J-YS were responsible for experimental design and data analysis. S-JK and H-GK performed the experiments, assembled and interpreted the data, and wrote the manuscript. Y-IP, HY and NT assisted with cell culture, $\mathrm{PCR}$ and immunostaining. S-RH was responsible for teratoma formation and animal care. All authors read and approved the final manuscript.

\section{Competing interests}

The authors declare that they have no competing interests.

Consent for publication

Not applicable.

Ethical approval and consent to participate

All animal experiments were performed according to the Code of Laboratory Animal Welfare Ethics, Animal and Plant Quarantine \& Inspection Agency $(\mathrm{Q} \mid \mathrm{A})$, Republic of Korea. All surgeries and sacrifices were performed under anesthesia. Every effort was made to minimize animal suffering.

Received: 3 September 2016 Revised: 9 February 2017

Accepted: 21 February 2017 Published online: 17 April 2017

\section{References}

1. Cai J, Zhao Y, Liu Y, Ye F, Song Z, Qin H, et al. Directed differentiation of human embryonic stem cells into functional hepatic cells. Hepatology. 2007:45(5):1229-39.

2. Yamamoto H, Quinn G, Asari A, Yamanokuchi H, Teratani T, Terada M, et al. Differentiation of embryonic stem cells into hepatocytes: biological functions and therapeutic application. Hepatology. 2003;37(5):983-93.

3. Hay DC, Zhao D, Fletcher J, Hewitt ZA, McLean D, Urruticoechea-Uriguen A, et al. Efficient differentiation of hepatocytes from human embryonic stem cells exhibiting markers recapitulating liver development in vivo. Stem Cells. 2008;26(4):894-902.

4. Si-Tayeb K, Noto FK, Nagaoka M, Li J, Battle MA, Duris C, et al. Highly efficient generation of human hepatocyte-like cells from induced pluripotent stem cells. Hepatology. 2010;51(1):297-305.

5. Touboul T, Hannan NR, Corbineau S, Martinez A, Martinet C, Branchereau S, et al. Generation of functional hepatocytes from human embryonic stem cells under chemically defined conditions that recapitulate liver development. Hepatology. 2010;51(5):1754-65

6. Shan J, Schwartz RE, Ross NT, Logan DJ, Thomas D, Duncan SA, et al. Identification of small molecules for human hepatocyte expansion and iPS differentiation. Nat Chem Biol. 2013;9(8):514-20.

7. Bone HK, Nelson AS, Goldring CE, Tosh D, Welham MJ. A novel chemically directed route for the generation of definitive endoderm from human embryonic stem cells based on inhibition of GSK-3. J Cell Sci. 2011;124(Pt 12):1992-2000.

8. Hay DC. Rapid and scalable human stem cell differentiation: now in 3D. Stem Cells Dev. 2013;22(20):2691-2.

9. Asplund A, Pradip A, van Giezen M, Aspegren A, Choukair H, Rehnstrom M, et al. One standardized differentiation procedure robustly generates homogenous hepatocyte cultures displaying metabolic diversity from a large panel of human pluripotent stem cells. Stem Cell Rev. 2016;12(1):90-104.

10. Fu W, Wang SJ, Zhou GD, Liu W, Cao Y, Zhang WJ. Residual undifferentiated cells during differentiation of induced pluripotent stem cells in vitro and in vivo. Stem Cells Dev. 2012;21(4):521-9.

11. Fujikawa T, Oh SH, Pi L, Hatch HM, Shupe T, Petersen BE. Teratoma formation leads to failure of treatment for type I diabetes using embryonic stem cell-derived insulin-producing cells. Am J Pathol. 2005;166(6):1781-91.

12. Teramoto K, Hara Y, Kumashiro Y, Chinzei R, Tanaka Y, Shimizu-Saito K, et al. Teratoma formation and hepatocyte differentiation in mouse liver transplanted with mouse embryonic stem cell-derived embryoid bodies. Transplant Proc. 2005:37(1):285-6.

13. Miura K, Okada Y, Aoi T, Okada A, Takahashi K, Okita K, et al. Variation in the safety of induced pluripotent stem cell lines. Nat Biotechnol. 2009;27(8):743-5.

14. Naujok O, Kaldrack J, Taivankhuu T, Jorns A, Lenzen S. Selective removal of undifferentiated embryonic stem cells from differentiation cultures through HSV1 thymidine kinase and ganciclovir treatment. Stem Cell Rev. 2010;6(3):450-61.

15. Schriebl K, Satianegara G, Hwang A, Tan HL, Fong WJ, Yang HH, et al. Selective removal of undifferentiated human embryonic stem cells using magnetic activated cell sorting followed by a cytotoxic antibody. Tissue Eng Part A. 2012;18(9-10):899-909.

16. Ben-David U, Gan QF, Golan-Lev T, Arora P, Yanuka O, Oren YS, et al. Selective elimination of human pluripotent stem cells by an oleate synthesis inhibitor discovered in a high-throughput screen. Cell Stem Cell. 2013;12(2):167-79.

17. Bieberich E, Silva J, Wang G, Krishnamurthy K, Condie BG. Selective apoptosis of pluripotent mouse and human stem cells by novel ceramide 
analogues prevents teratoma formation and enriches for neural precursors in ES cell-derived neural transplants. J Cell Biol. 2004;167(4):723-34.

18. Lee MO, Moon SH, Jeong HC, Yi JY, Lee TH, Shim SH, et al. Inhibition of pluripotent stem cell-derived teratoma formation by small molecules. Proc Natl Acad Sci U S A. 2013;110(35):E3281-90.

19. Rauch A, Hennig D, Schafer C, Wirth M, Marx C, Heinzel T, et al. Survivin and YM155: how faithful is the liaison? Biochim Biophys Acta. 2014;1845(2):202-20.

20. Kang SJ, Lee HM, Park YI, Yi H, Lee H, So B, et al. Chemically induced hepatotoxicity in human stem cell-induced hepatocytes compared with primary hepatocytes and HepG2. Cell Biol Toxicol. 2016;32(5):403-17.

21. Kang SJ, Park YI, Kwon MJ, Yang YH, Bang SI, Sohn SH, Park YH, So B, Kang HG. Adipose stromal cells are a more efficient source than adipose stem cells in retrovirus-mediated iPS induction. Cell Mol Bioeng. 2015;8(1):224-35.

22. Bieberich E. Smart drugs for smarter stem cells: making SENSe (sphingolipid-enhanced neural stem cells) of ceramide. Neurosignals. 2008; 16(2-3):124-39.

23. DeLaForest A, Nagaoka M, Si-Tayeb K, Noto FK, Konopka G, Battle MA, et al. HNF4A is essential for specification of hepatic progenitors from human pluripotent stem cells. Development. 2011;138(19):4143-53.

24. Hay DC, Fletcher J, Payne C, Terrace JD, Gallagher RC, Snoeys J, et al. Highly efficient differentiation of hESCs to functional hepatic endoderm requires ActivinA and Wnt3a signaling. Proc Natl Acad Sci U S A. 2008;105(34):12301-6.

25. Ogawa S, Surapisitchat J, Virtanen C, Ogawa M, Niapour M, Sugamori KS, et al. Three-dimensional culture and CAMP signaling promote the maturation of human pluripotent stem cell-derived hepatocytes. Development. 2013;140(15):3285-96

26. Godoy P, Hewitt NJ, Albrecht U, Andersen ME, Ansari N, Bhattacharya S, et al. Recent advances in $2 \mathrm{D}$ and $3 \mathrm{D}$ in vitro systems using primary hepatocytes, alternative hepatocyte sources and non-parenchymal liver cells and their use in investigating mechanisms of hepatotoxicity, cell signaling and ADME. Arch Toxicol. 2013;87(8):1315-530.

27. Lee AS, Tang C, Cao F, Xie X, van der Bogt K, Hwang A, et al. Effects of cell number on teratoma formation by human embryonic stem cells. Cell Cycle. 2009;8(16):2608-12.

\section{Submit your next manuscript to BioMed Central and we will help you at every step:}

- We accept pre-submission inquiries

- Our selector tool helps you to find the most relevant journal

- We provide round the clock customer support

- Convenient online submission

- Thorough peer review

- Inclusion in PubMed and all major indexing services

- Maximum visibility for your research

Submit your manuscript at www.biomedcentral.com/submit

) Biomed Central 